\title{
BMJ Open Assessment tools for the measurement of the self-efficacy of drug users: protocol for a systematic review
}

\author{
Selene Cordeiro Vasconcelos, ${ }^{1}$ Iracema da Silva Frazão, ${ }^{2}$ Everton Botelho Sougey, ${ }^{3}$ \\ Sandra Lopes de Souza, ${ }^{4}$ Tatiana de Paula Santana da Silva, ${ }^{3}$ \\ Murilo Duarte da Costa Lima
}

To cite: Vasconcelos SC, Frazão IdS, Sougey EB, et al. Assessment tools for the measurement of the self-efficacy of drug users: protocol for a systematic review. BMJ Open 2018;8:e019019. doi:10.1136/ bmjopen-2017-019019

- Prepublication history for this paper is available online. To view these files, please visit the journal online (http://dx.doi. org/10.1136/bmjopen-2017019019).

Received 21 August 2017

Revised 4 January 2018

Accepted 8 January 2018

\section{Check for updates}

${ }^{1}$ Department of Nursing in Public Health, Federal University of Paraíba, João Pessoa, Paraíba, Brazil

${ }^{2}$ Department of Nursing, Federal University of Pernambuco,

Recife, Brazil

${ }^{3}$ Department of Neuropsychiatry, Federal University of

Pernambuco, Recife, Brazil

${ }^{4}$ Department of Anatomy,

Federal University of

Pernambuco, Recife, Brazil

Correspondence to

Prof Selene

Cordeiro Vasconcelos;

selumares@yahoo.com.br

\section{ABSTRACT}

Introduction The abuse of alcohol and other drugs is a worldwide problem, the treatment of which poses a challenge to healthcare workers.

Objective This study presents a proposal for a systematic review to analyse the psychometric properties of assessment tools developed to measure the self-efficacy of drug users with regard to resisting the urge to take drugs in high-risk situations.

Methods and Analysis The guiding question was based on PICOS (Population Intervention Comparator Outcome Setting), and the report of the methods of review protocol was written in accordance with the Preferred Reporting Items for Systematic Review and Meta-Analysis Protocols (PRISMA-P). Searches will be performed in the PsycINF0, Cochrane, Pubmed, Web of Science, SCOPUS and CINAHL databases, followed by the use of the 'snowball' strategy. The inclusion criteria for the articles will be (1) assessment tool validation studies; (2) assessment tools developed to measure self-efficacy; (3) quantitative measures; (4) measures designed for use on adults; (5) data from self-reports of the participants; (6) studies involving a description of psychometric properties of the measures; and (7) studies that explain how the level of self-efficacy is scored. The search, selection and analysis will be performed by two independent reviewers. In cases of a divergence of opinion, a third reviewer will be consulted. The COSMIN checklist will be used for the appraisal of the methodological quality of the assessment tools and the certainty of the evidence in the articles (risk of bias) will be analysed using the GRADE (Grading of Recommendations Assessment, Development and Evaluation) approach.

Ethics and dissemination This protocol does not require ethical approval. However, this protocol is part of the thesis entitled Drug-Taking Confidence Questionnaire for use in Brazil, presented for obtaining a doctorate in neuropsychiatry and behavioural sciences from the Federal University of Pernambuco, and has received approval from the human research ethics committee of the Federal University of Pernambuco (reference number: 1.179.162). The results will be disseminated to clinicians and researchers through peer-reviewed publications and conferences.

PROSPERO registration number CRD42017068555.

\section{Strengths and limitations of this study}

- The article will recommend a gold standard among existing assessment tools for the measurement of self-efficacy related to resisting the urge to take drugs in high-risk situations.

- The study will involve the use of quantitative methods for appraising the strength of the evidence encountered.

- This will be the first review on assessment tools for measuring self-efficacy related to resisting the urge to take drugs in high-risk situations.

- The study will be developed at a single research centre.

Grey literature will not be included.

\section{BACKGROUND}

Dependence on alcohol and other drugs is characterised by behaviour aimed at maintaining use as well as the loss of pleasure in habitual activities. It is a maladaptive way to cope with stressful situations and is considered a serious public health problem throughout the world. $^{1-3}$ Cognitive and behavioural alterations are among the harmful effects of substance abuse, ${ }^{4-6}$ affecting personal, familial and social relations as well as compromising an individual's self-efficacy with regard to resisting the urge to take drugs in high-risk situations. $^{\text {? }}$

Bandura $(1977)^{8}$ conceives self-efficacy as a belief or personal confidence in one's ability to perform a specific action for one's own benefit. Thus, self-efficacy is a mental process that guides behaviour and exerts an influence on the establishment of goals, one's motivation level, perseverance in the presence of setbacks and resilience in the face of adversity. ${ }^{8-11}$

Different subtypes of self-efficacy are described in the literature ${ }^{12}$ and several assessment tools have been developed to measure this construct among individuals who are 
dependent on alcohol ${ }^{13-16}$ and/or other drugs, ${ }^{17-21}$ and in situations of combined use. ${ }^{22-25}$

Self-efficacy with regard to resisting the urge to take drugs in high-risk situations is considered a strong predictor of abstinence or a reduction in drug use and is related to the results of treatment. ${ }^{26-28}$ Considering the importance of this subtype, the number of assessment tools developed to measure this phenomenon and the lack of recommendations regarding the most robust assessment tools, there is a need to evaluate the psychometric properties of available measures and recommend an assessment tool that can serve as the gold standard.

The proposed systematic review will be able to assist healthcare professionals in the choice of the most adequate assessment tools for their clinical practice with the aim of monitoring levels of self-efficacy to resist the urge to take drugs in high-risk situations. ${ }^{29}$ The guiding question of the study will be 'Do assessment tools designed to measure self-efficacy with regard to resisting the urge to take drugs in high-risk situations have adequate psychometric properties?'.

Thus, the aim of this protocol is to propose a systematic review to analyse the psychometric properties of assessment tools developed to measure the self-efficacy of drug users to resist the urge to consume these substances in high-risk situations.

\section{METHOD/DESIGN}

\section{Design and registration of the study}

This proposal for a systematic review is registered with the International Prospective Registry of Systematic Reviews (PROSPERO) in CRD 42017068555 (https:// www.crd.york.ac.uk/PROSPERO/register_new_review. asp). The report of the methods of review protocol was written in accordance with the Preferred Reporting Items for Systematic Review and Meta-Analysis Protocols (PRISMA-P) ${ }^{30}$ The report of the methods of the systematic review article will follow the guidelines of PRISMA. ${ }^{31}$

\section{Inclusion of articles}

All methodological articles developed for the validation of assessment tools with a quantitative approach for adult drug users ( $\geq 18$ years of age) based on self-reported data and that describe psychometric properties, the clinical usefulness of which consists of the measurement of self-efficacy in users of alcohol and/or other drugs with regard to resisting the urge to use such substances in high-risk situations, will be included. No restrictions will be imposed with regard to language or publication date. Review studies will be excluded.

\section{Search strategy}

The guiding question was based on the PICOS strategy ${ }^{32}$ (Population Intervention Comparator Outcome Setting). Electronic searches will be conducted in the PsycINFO, Cochrane, Pubmed, Web of Science, SCOPUS and CINAHL databases. After the retrieval of articles from the databases, the snowball strategy will be employed. ${ }^{33}$ Grey literature will not be considered.

To reduce the risk of bias in this step, two independent reviewers will perform the searches and preselect articles based on an analysis of the titles and abstracts for potentially eligible articles and assessment tools. Preselected articles will be submitted to full-text analysis for the determination of the studies that will make up the final sample. The level of agreement between the two reviewers will be calculated. In cases of divergences of opinion, the reviewers will discuss the article in question until reaching a consensus. A third reviewer will be consulted, if necessary.

The entire process will be stored in a data bank to ensure access to the records of the initial search strategy, the snowball strategy as well as the excluded articles and the reasons for exclusion. Duplicate articles will only be counted once. The following MeSH terms and combinations will be employed in the searches: 'self-efficacy', 'coping', 'validation studies', 'drug users', 'scale', 'instrument', 'questionnaire' and 'outcome assessment'. Adjustments to the keywords may be made during the execution of the systematic review.

\section{Tracking, data extraction and content comparison analysis} The data extracted from the articles selected will be organised on a chart specifically designed for the systematic review, which will contain the following:

- General characteristics of the study: Authors, date of publication, country of origin, objective, sample size and main outcomes.

- Description of assessment tools: Name and acronym; objective; domains, dimensions or subscales; description of high-risk situations; number of items; method of collecting self-reported data; description of scoring and classification of levels of self-efficacy; administration method; cut-off points; and psychometric properties validated by the authors.

When necessary, the author of the articles and assessment tools will be contacted to obtain further information.

A flowchart will be created illustrating the selection and analysis methods. Relevant data from all articles will be summarised in tables and/or charts. Thus, the systematic review will offer a general overview of all available instruments for measuring the self-efficacy of drug users for resisting the urge to take these substances in high-risk situations.

\section{Appraisal of methodological quality of selected articles and measures}

To evaluate the risk of bias, the articles included in the final sample will be analysed with regard to methodological quality and the strength or certainty of the evidence offered using the GRADE approach (Grading of Recommendations Assessment, Development and Evaluation). ${ }^{34}$

The appraisal of the methodological quality of the assessment tools will follow the COSMIN 
(COnsensus-based Standards for the selection of health Measurement INstruments) criteria, using only the A-H boxes on the checklist to rate the quality of each property. ${ }^{35}$ The checklists for interpretability and generalisation will not be used because these lists are only related to data extraction.

The 4-point COSMIN scoring system will be used to classify the assessment tools as excellent (adequate methodological quality), good (missing information, but quality could be considered fair) or poor (inadequate quality). Assessment tools with varied results (some points considered excellent and others considered poor) will be classified based on the lower scores. ${ }^{35-39}$ Two reviewers will analyse the risk of bias and classify the assessment tools in an independent manner.

\section{Evaluation of clinical usefulness of assessment tools}

The analysis of clinical usefulness will follow the criteria proposed by Tyson and Brown (2014) ${ }^{40}$ related to interpretability and viability, with the aim of quantifying the practical aspects of the measures based on factors that can influence the decision-making process of health professionals in clinical practice. ${ }^{41}$ These criteria are listed below:

- Total time required for the administration, analysis and interpretation of the data obtained using the measure: $<10 \mathrm{~min}$ (three points); $10-30 \mathrm{~min}$ (two points); $30-60 \mathrm{~min}$ (one point) and $>1$ hour (zero points).

- Cost of assessment tool: < $£ 100$ (3 points); £100500 (two points); $£ 500-1000$ (one point); $£ 1000$ (zero points).

- Need for specialised equipment and training for use: none (two points); yes, but simple and clinically viable (one point); yes and not clinically viable/unknown (zero points.

- Portability of the assessment tool (can it be taken to the patient?): yes, easily (fits in pocket) (two points); yes (fits in a carrying case) (one point); no or very difficult (zero points).

- Accessibility of the tool (are detailed instructions for use available?): yes (complete operating procedure/ instruction manual can be obtained in article or site) (two points); no, but the operation can be performed simply based on the description in the article (one point); no available instructions for use (zero points).

\section{Data synthesis}

The assessment tools will be described in tables and/ or charts highlighting the general characteristics, application contexts, applicability and information on the evaluation methods of the measures. At the end of the analyses, assessment tools with the following qualities will be considered adequate for measuring self-efficacy with regard to resisting the urge to consume drugs in high-risk situations:

- Those with a methodology considered 'good' or 'excellent' based on the COSMIN checklist; ${ }^{35-39}$
- Those with a score of 10 or more points on the clinical usefulness evaluation scale proposed by Tyson and Brown (2014). ${ }^{40}$

\section{DISCUSSION}

Special care will be taken regarding the storage and adequate use of the data produced in this study. Self-efficacy is considered an important component of the treatment process for drug users and many assessment tools have been developed to measure this phenomenon, which justifies the need to identify which of these assessment tools could be considered the gold standard for this purpose.

The proposed study will present the psychometric data of assessment tools developed to measure self-efficacy with regard to resisting the urge to take drugs in highrisk situations in order to identify a gold standard for the analysis of this construct.

Therefore, the proposed review will investigate the psychometric properties and clinical usefulness of assessment tools developed to measure the self-efficacy of drug users with regard to resisting the urge to take drugs in high-risk situations. The aim is to recommend a gold standard among the different assessment tools used to measure self-efficacy in this context and offer a discussion on the strong points and limitations of the measures through an analysis of the general characteristics, psychometric properties and clinical usefulness of the measures as well as the methodological quality of the studies.

The review intends to be clear and specific with regard to methodological rigour, employing a replicable systematic approach for the search strategy, screening, evaluation and data extraction of the studies retrieved from the available databases. Validated instruments for measuring given phenomena, such as self-efficacy, offer valid and reliable results that can guide health professionals with regard to interventions for drug users and assist in the adoption of adequate strategies for the promotion of self-efficacy and the minimisation of the harm caused by substance abuse.

Contributors All authors made substantial contributions to the concept and study design and participated in the drafting of the submission request. SCV and TPSS conceived the study, developed the inclusion criteria, performed the search and selection of the studies, and wrote the present systematic review protocol article. ISF, EBS, SLS and MDCL guided all phases of this systematic review protocol article and performed a critical review of the manuscript. All authors read and approved the final version.

Funding This research received no specific grant from any funding agency in the public, commercial or not-for-profit sectors.

Competing interests None declared.

Patient consent Not required.

Provenance and peer review Not commissioned; externally peer reviewed.

Open Access This is an Open Access article distributed in accordance with the Creative Commons Attribution Non Commercial (CC BY-NC 4.0) license, which permits others to distribute, remix, adapt, build upon this work non-commercially, and license their derivative works on different terms, provided the original work is properly cited and the use is non-commercial. See: http://creativecommons.org/ licenses/by-nc/4.0/ 
(c) Article author(s) (or their employer(s) unless otherwise stated in the text of the article) 2018. All rights reserved. No commercial use is permitted unless otherwise expressly granted.

\section{REFERENCES}

1. Nakhli J, Gorsane MA, Bouhlel S, et al. [Prevalence of alcoholism in primary care in the governorate of Sousse]. Tunis Med 2015:93:297-301.

2. Lubman DI, Garfield JB, Manning V, et al. Characteristics of individuals presenting to treatment for primary alcohol problems versus other drug problems in the Australian patient pathways study. BMC Psychiatry 2016;16:250.

3. Koob GF. Antireward, compulsivity, and addiction: seminal contributions of Dr. Athina Markou to motivational dysregulation in addiction. Psychopharmacology 2017;234:1315-32.

4. Almeida RMM, Flores ACS, Scheffer M. Suicidal ideation, problem solving, anger expression and impulsivity in psychoactive substance dependents. Psicologia: Reflexão \& Critica 2013;26:p1.

5. Silva ER, Ferreira ACZ, Borba LO, et al. Impact of drugs on the physical and mental health of dependents. Cienc Cuid Saude 2016;15:101-8.

6. Peuker AC, Lopes FM, Menezes CB, et al. Implicit processing and chemical dependence: theory, assessment and perspectives. Psicologia: Teoria e Pesquisa 2013;29:7-14.

7. Pirnia B, Tabatabaei SK, Tavallaii A, et al. The efficacy of contingency management on cocaine craving, using prize-based reinforcement of abstinence in cocaine users. Electron Physician 2016;8:3214-21.

8. Bandura A. Self-efficacy: toward a unifying theory of behavioral change. Psychol Rev 1977;84:191-215.

9. Bandura A. A sociocognitive analysis of substance abuse: an agentic perspective. Psychol Sci 1999;10:214-7.

10. Bandura A, Azzi RG, Polydoro S. Teoria social cognitiva: conceitos básicos. Porto Alegre: Artmed, 2008:97-114.

11. Bandura A. Health promotion by social cognitive means. Health Educ Behav 2004;31:143-64.

12. Diclemente CC, Fairhurst SK, Piotrowski NA. Self-efficacy and addictive behaviors. In: Maddux JE, ed. Self-efficacy, adaptation and adjustment: theory, research and application. New York: Plenum Press, 1995:109-41.

13. Young RM, Oei TPS, Crook GM. Development of a drinking selfefficacy questionnaire. J Psychopathol Behav Assess 1991;13:1-15.

14. DiClemente CC, Carbonari JP, Montgomery RP, et al. The alcoho abstinence self-efficacy scale. J Stud Alcohol 1994;55:141-8.

15. Kraus SW, Rosenberg H, Bonar EE, et al. Assessing self-efficacy to reduce one's drinking: further evaluation of the alcohol reduction strategies-current confidence questionnaire. Alcohol Alcohol 2012;47:312-6.

16. Martin GW, Wilkinson DA, Poulos CX. The drug avoidance selfefficacy scale. J Subst Abuse 1995;7:151-63.

17. De Weert-Van Oene GH, Breteler MH, Schippers GM, et al. The validity of the Self-Efficacy List for Drug Users (SELD). Addict Behav 2000;25:599-605.

18. Phillips KT, Rosenberg $\mathrm{H}$. The development and evaluation of the Harm Reduction Self-Efficacy Questionnaire. Psychol Addict Behav 2008;22:36-46.

19. Parr JM, Kavanagh DJ, Young RM, et al. Development of self-efficacy and expectancy measures for benzodiazepines. Addict Behav 2009;34:751-6.

20. Minervini I, Palandri S, Bianchi S, et al. Desire and Coping SelfEfficacy as Craving Measures in Addiction: The Self-Efficacy and Desire Scale (SAD). The Open Behavioral Science Journal 2011;5:1-7.

21. Sklar SM, Annis HM, Turner NE. Development and validation of the drug-taking confidence questionnaire: a measure of coping selfefficacy. Addict Behav 1997;22:655-70.
22. Turner NE, Annis HM, Sklar SM. Measurement of antecedents to drug and alcohol use: psychometric properties of the Inventory of Drug-Taking Situations (IDTS). Behav Res Ther 1997;35:465-83.

23. Sklar SM, Turner NE. A brief measure for the assessment of coping self-efficacy among alcohol and other drug users. Addiction 1999;94:723-9.

24. Miller PJ, Ross SM, Emmerson RY, et al. Self-efficacy in alcoholics: clinical validation of the Situational Confidence Questionnaire. Addict Behav 1989;14:217-24.

25. Clingan SE, Woodruff SI. Drug-avoidance self-efficacy among exclusive cannabis users vs. other drug users visiting the emergency department. Subst Use Misuse 2017:52:1222-8.

26. Majer JM, Chapman HM, Jason LA. Abstinence self-efficacy and substance use at 2 years: the moderating effects of residential treatment conditions. Alcohol Treat Q 2016;34:386-401.

27. Shrestha R, Altice FL, Huedo-Medina TB, et al. Willingness to use Pre-Exposure Prophylaxis (PrEP): an empirical test of the Information-Motivation-Behavioral Skills (IMB) model among highrisk drug users in treatment. AIDS Behav 2017;21:1299-308.

28. Hughes JR, Naud S. Perceived role of motivation and self-efficacy in smoking cessation: A secondary data analysis. Addict Behav 2016;61:58-61.

29. Stevens AK, Littlefield AK, Blanchard BE, et al. Does drinking refusal self-efficacy mediate the impulsivity-problematic alcohol use relation? Addict Behav 2016;53:181-6.

30. Moher D, Shamseer L, Clarke M, et al. Preferred reporting items for systematic review and meta-analysis protocols (PRISMA-P) 2015 statement. Syst Rev 2015;4:1-9.

31. Liberati A, Altman DG, Tetzlaff J, et al. The PRISMA statement for reporting systematic reviews and meta-analyses of studies that evaluate health care interventions: explanation and elaboration. PLoS Med 2009;6:e1000100

32. University of York. Centre for reviews and dissemination. Systematic reviews: CRD's guidance for undertaking reviews in health care. York, UK: University of York, 2009.

33. van Weert JCM, van Munster BC, Sanders R, et al. Decision aids to help older people make health decisions: a systematic review and meta-analysis. BMC Med Inform Decis Mak 2016;16:1-20.

34. Atkins D, Best D, Briss PA, et al. Grading quality of evidence and strength of recommendations. BMJ 2004;328:1490-4.

35. Mokkink LB, Terwee CB, Patrick DL, et al. The COSMIN checklist for assessing the methodological quality of studies on measurement properties of health status measurement instruments: an international Delphi study. Qual Life Res 2010;19:539-49.

36. Terwee CB, Mokkink LB, Knol DL, et al. Rating the methodological quality in systematic reviews of studies on measurement properties: a scoring system for the COSMIN checklist. Qual Life Res 2012;21:651-7.

37. Mokkink LB, Terwee CB, Patrick DL, et al. The COSMIN study reached international consensus on taxonomy, terminology, and definitions of measurement properties for health-related patientreported outcomes. J Clin Epidemiol 2010;63:737-45.

38. Mokkink LB, Terwee CB, Gibbons E, et al. Inter-rater agreement and reliability of the COSMIN (COnsensus-based Standards for the selection of health status Measurement Instruments) checklist. BMC Med Res Methodol 2010;10:82.

39. Mokkink LB, Terwee CB, Stratford PW, et al. Evaluation of the methodological quality of systematic reviews of health status measurement instruments. Qual Life Res 2009;18:313-33.

40. Tyson SF, Brown P. How to measure fatigue in neurological conditions? A systematic review of psychometric properties and clinical utility of measures used so far. Clin Rehabil 2014;28:804-16.

41. Martins JC, Aguiar LT, Nadeau S, et al. Measurement properties of self-report physical activity assessment tools in stroke: a protocol for a systematic review. BMJ Open 2017;7:e012655-5. 